\title{
F-box Protein $\beta$ TrCP1 Is a Substrate of Extracellular Signal-regulated Kinase 2
}

\author{
Cheol-Jung Lee ${ }^{1,2, *}$, Ga-Eun Lee ${ }^{1, *}$, Hyun-Jung An', Eun Suh $\mathrm{Cho}^{3}$, Weidong Chen', Joo Young Lee', \\ Han Chang Kang', Hye Suk Lee', Yong-Yeon Cho' \\ ${ }^{1}$ BRL \& BK21-4th Team, College of Pharmacy, The Catholic University of Korea, Bucheon, ${ }^{2}$ Research Center for Materials \\ Analysis, Korea Basic Science Institute, Daejeon, Korea, ${ }^{3}$ Department of Biochemistry, College of Biological Sciences, \\ University of Minnesota, Minneapolis, MN, USA
}

F-box proteins, consisting of 69 members which are organized into the three subclasses FBXW, FBXL, and FBXO, are the substrate specific recognition subunits of the SKP1-Cullin 1-F-box protein E3 ligase complex. Although $\beta$ TrCP 1 and 2, members of the FBXW subfamily, are known to regulate some protein stability, molecular mechanisms by which these proteins can recognize proper substrates are unknown. In this study, it was found that $\beta \operatorname{TrCP} 1$ showed strong interaction with members of mitogen-activated protein kinases. Although extracellular signal-regulated kinase (ERK) 3, p38 $\beta$, and p38 $\delta$ showed weak interactions, ERK2 specifically interacted with $\beta \mathrm{TrCP} 1$ as assessed by immunoprecipitation. In interaction domain determination experiments, we found that ERK2 interacted with two independent ERK docking sites located in the F-box domain and linker domain, but not the WD40 domain, of $\beta \operatorname{TrCP} 1$. Notably, mutations of $\beta \operatorname{TrCP} 1$ at the ERK docking sites abolished the interaction with ERK2. $\beta$ TrCP1 underwent phosphorylation by EGF stimulation, while the presence of the mitogen-activated protein kinase kinases inhibitor U0126, genetic silencing by sh-ERK2, and mutation of the ERK docking site of $\beta \operatorname{TrCP} 1$ inhibited phosphorylation. This inhibition of $\beta$ TrCP1 phosphorylation resulted in a shortened half-life and low protein levels. These results suggest that ERK2-mediated $\beta$ TrCP1 phosphorylation may induce the destabilization of $\beta \operatorname{TrCP} 1$.

Key Words Beta transducin repeat-containing protein, ERK pathway, Protein interaction domains, Binding site, Phosphorylation

\section{INTRODUCTION}

Extracellular stimuli, including growth factors (e.g. EGF), cytokines, and environmental stresses (e.g. ultraviolet), activate the receptor tyrosine kinase at the cytoplasmic membrane by dimerization-mediated autophosphorylation at the cytoplasmic domain [1]. The phosphorylated tyrosine residues of EGF receptor act as an initiation signal to recruit the Shc- Growth factor receptor-bound protein 2 (Grb2)-Ras complex and phospholipase $\mathrm{C}_{\gamma}$ in the cytoplasm, resulting in Son of sevenless-mediated Ras activation and intracellular $\mathrm{Ca}^{2+}$-mediated protein kinase $\mathrm{C}$ activation [2]. Activated Ras induced activation of the signaling axis of Rafs, mitogen-activated protein kinase kinases (MEKs), extracellular signal-regulated kinases (ERKs), and p90 ribosomal S6 kinase (RSKs), including RSK2 which regulates cell proliferation, carcinogenesis, ad- aptation, apoptosis [3], and protein stability regulation [4].

ERK1 and ERK2 are downstream kinases of MEKs and approximately share an $85 \%$ amino acid similarity. ERKs activated by MEKs-mediated direct phosphorylation at Thr and Tyr residues in the activation loop phosphorylate or interact with a large number of cytosolic and nuclear substrates specifically involved in cellular signaling of transcription factors responsible for particular cellular processes [5]. Thus, dysregulation of the aforementioned cascade frequently leads to the development of diverse diseases, including $>90 \%$ of all cancers [6]. Due to the importance of ERK as an intermediary signaling molecules, its activity must be precisely regulated. Our previous study demonstrated that ERK 1 or 2 take part in forming the $\mathrm{SCF}^{\beta \mathrm{TTCP} 1}$ complex that plays a key role in regulating ubiquitination-mediated protein stability, with Kruppel-like factor 4 (KIf4) [4]. While ERK 1- and 2-mediated

Received August 24, 2021, Revised September 10, 2021, Accepted September 13, 2021

Correspondence to Yong-Yeon Cho, E-mail: yongyeon@catholic.ac.kr, https://orcid.org/0000-0003-1107-2651

Check for updates

*These authors contributed equally to this study. 
cellular signaling pathways have been studied mainly with focus on phosphorylation-mediated transient activity regulation, the ERK 1 and 2 protein stability regulation has been poorly understood.

$\beta \operatorname{TrCP} 1$ and 2 are members of F-box proteins, which confer substrate selectivity for ubiquitination. F-box proteins are currently classified according to their potential roles, such as tumor suppressors (FBXW7, FBXO11, FBXW8, FBXL3, FBXO1, FBXO4, and FBXO18), oncogenes (SKP2, FBXO5, and $\mathrm{FBXO9}$ ), and context-dependent different functions $(\beta \operatorname{TrCP} 1$ and $\beta \operatorname{TrCP} 2)[7,8]$. Accumulating data suggest that the roles of $\beta \operatorname{TrCP} 1$ and 2 in cellular phenotypes are context dependent [7]. Ectopic expression of $\beta \operatorname{TrCP} 1$ controlled under the mouse mammary tumor virus promoter developed tumors such as mammary, ovarian, and uterine carcinoma in about 38\% transgenic mice [9]. Moreover, high $\beta$ TrCP1 mRNA and protein levels were detected in about $56 \%$ of colorectal cancer tissues with poor clinical prognosis [10]. In addition, somatic mutations of $\beta \operatorname{TrCP} 1$ and $\beta \operatorname{TrCP} 2$ identified in human gastric cancer correlated with stabilization of $\beta$-catenin which could explain the tumor development $[11,12]$. This highlights the tumor-suppressive role of $\beta$ TRCP 1 and 2 in the gastric cancer setting [7]. Moreover, various $\beta$ TRCPs substrates are known to have oncogenic properties. These include $\beta$-catenin, CDC25A, FBXO5, VEGF receptor 2, inhibitor of nuclear factor- $\kappa \mathrm{B}\left(\mathrm{l}_{\kappa} \mathrm{B}\right)$, programmed cell death protein 4 , and DEP domain-containing mTOR-interacting protein [7]. Since $\beta$ TRCP 1 and 2 might be involved in diverse cellular processes in accordance with substrate diversity, existence of other substrates of the $\beta$ TRCP 1 and 2 may be possible.

In our present study, ERK2 was found to be a binding partner of $\beta$ TRCP1. It was discovered that the binding is mediated through the $\mathrm{D}, \mathrm{F}$-box, and linker domains of $\beta$ TRCP1, not the WD40 domain. It was further found that two putative ERK docking sites (PEDSs) were identified at the F-box and linker regions. Notably, the binding of $\beta$ TRCP1 to ERK2 was reduced by the mutations at these sites. Taken together, these results suggested that interaction between ERK2 and $\beta$ TRCP1, not ERK ubiquitination, may regulate the activity of $\beta$ TRCP1.

\section{MATERIALS AND METHODS}

\section{Reagents and antibodies}

Chemicals for molecular and cell biology purposes were purchased from Sigma-Aldrich (St. Louis, MO, USA). Dulbecco's modified Eagle's medium (DMEM) was obtained from Corning (Cat. \#: 10-013-CVR, New York, NY, USA). The supplements for cell culture, including penicillin/streptomycin (Cat. \#: 15140-122) and sodium pyruvate (100×, Cat. \#: 11360070), were purchased from Life Science Technologies (Rockville, MD, USA). FBS (Cat. \#: 26140-079) was purchased from Corning. Antibodies (Abs) against phospho-MEK1/2 (S217/221) (p-MEKs, Cat. \#: 9154), phospho-ERK1/2 (T202,
Y204) (p-ERKs, Cat. \#: 9106), total-MEK1/2 (t-MEKs, Cat. \#: 4694), and total-ERK1/2 (t-ERKs, Cat. \#: 9102) were purchased from Cell Signaling Technology (Beverly, MA, USA). The $\beta$-actin antibody (Cat. \#: SC-69879) was purchased from Santa Cruz Biotechnology (Santa Cruz, CA, USA). Dimethylsulfoxide (DMSO, Cat. \#: D8418) was purchased from Sigma-Aldrich. U0126, an MEK inhibitor (Cat. \#: 9903, Cell Signaling Biotechnology), was dissolved in DMSO to obtain stock solutions $(\times 1,000$ high concentration).

\section{Cell culture}

HEK293T cell line was purchased from the American Type Culture Collection (Manassas, VA, USA). The HEK293T cells were cultured in DMEM supplemented with $10 \% \mathrm{FBS}$ and antibiotics. The cells were maintained at $37^{\circ} \mathrm{C}$ in a $5 \% \mathrm{CO}_{2}$ incubator and were split at $90 \%$ confluence. The medium was exchanged every 2 or 3 days. When the cells reached $60 \%$ confluence, they were transfected with expression vectors by using jetPEI (Polyplus-Transfection, New York, NY, USA) according to the manufacturer's instructions.

\section{Vector construction}

The $\beta$ TrCP1 open reading frame was incorporated into multicloning sites of the pACT-VP16 mammalian two-hybrid vector. The open reading frame of kinases was amplified from the human kinase open reading frame kit, containing 556 distinct human kinases and kinase-related protein open reading frames in pDONR-223 Gateway ${ }^{\circledR}$ Entry vectors (Addgene, Watertown, MA, USA), and inserted into the pBINDGal4 mammalian two hybrid vector. For transient transfection of $\beta$ TrCP1, Cullines, ERK2, and truncated and serial deleted $\beta$ TrCP, mammalian expression vectors pcDNA3-HA, pcDNA3-Myc, and pcDNA4-HisMax-Awere utilized. All recombined expression vectors were confirmed by DNA sequencing.

\section{Mammalian two-hybrid assay}

To screen for protein-binding partners, we conducted mammalian two-hybrid assays in accordance with the Promega Checkmate mammalian two-hybrid system protocols (Promega, Madison, WI, USA). HEK293T cells $\left(2 \times 10^{4}\right.$ cells/well $)$ were seeded into 48-well plates and maintained with $10 \%$ FBS-DMEM for 18 hours before conducting transfection. The vectors pACT-VP16- $\beta$ TrCP1, pBIND-Gal4-kinases, and pG5-luciferase were mixed at the same molar ratios (1:1:1) and the total amount of DNA was no more than $100 \mathrm{ng}$ per well. Transfection was done utilizing jetPEI according to the manufacturer's recommendations. For the luciferase assay, the cells were disrupted by directly adding a cell lysis buffer and gently shaking for 30 minutes at room temperature. Then, $60 \mu \mathrm{L}$ aliquot was added to each-well luminescence plate. Luminescence activity was automatically measured through a VICTOR X3 plate reader (PerkinEImer, Waltham, MA, USA). To evaluate transfection efficiency, relative lucif- 
erase activity was calculated based on the pG5-luciferase basal control and was normalized against Renilla luciferase activity, which was included in the pBIND vector.

\section{Gene silencing}

To silence $\beta$ TrCP1 or ERK2 in HEK293T, lentiviral expression plasmids of pLenti-sh-ERK2 (Dharmacon, Lafayette, CO, USA) were co-transfected into HEK293T cells with psPAX2 and pMD2.G (Addgene, Cambridge, MA, USA) as indicated by the manufacturer's recommended protocols. At 24 hours and 48 hours after transfection, we obtained a Lenti-sh-ERK2 medium containing viral particles from the HEK293T cells. The medium was filtered with a $0.45 \mu \mathrm{m}$ filter (Cat. \#: 7232545, Thermo Fisher Scientific, Waltham, MA, USA) and used, with 1 to $2 \mu \mathrm{g} / \mathrm{mL}$ of polybrene, to infect HEK293T. Af- ter a maximum of 16 hours, the cell medium was exchanged with fresh complete medium. After 48 hours of maintenance, non-infected control cells were killed over a period of 3 days by treatment with $2 \mu \mathrm{g} / \mathrm{mL}$ of puromycin (Cat. \#: A111308, Thermo Fisher Scientific). Surviving cells were immediately examined to determine protein levels by Immunoprecipitation (IP) and Western blotting.

\section{Western blot analysis}

Samples containing equal amounts of proteins (30 to 50 $\mu \mathrm{g})$ were resolved by $8 \%$ to $10 \%$ SDS PAGE 1 and then transferred onto polyvinylidene difluoride membranes. The membranes were blocked in 5\% skim milk and hybridized with specific primary and HRP-conjugated secondary Abs as indicated. The membranes were washed, and target proteins

\section{A}

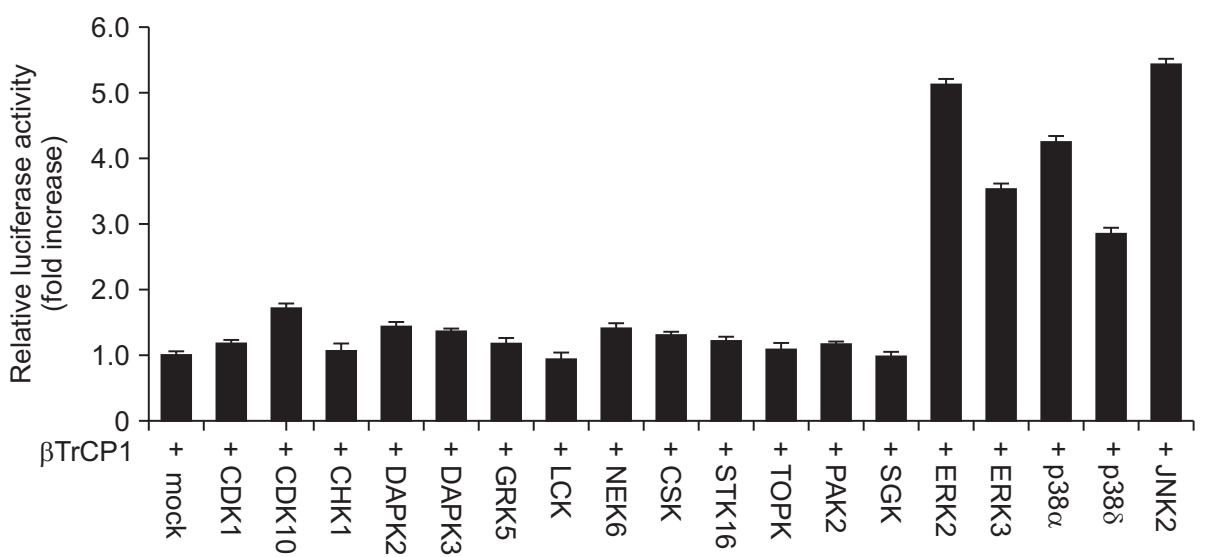

B

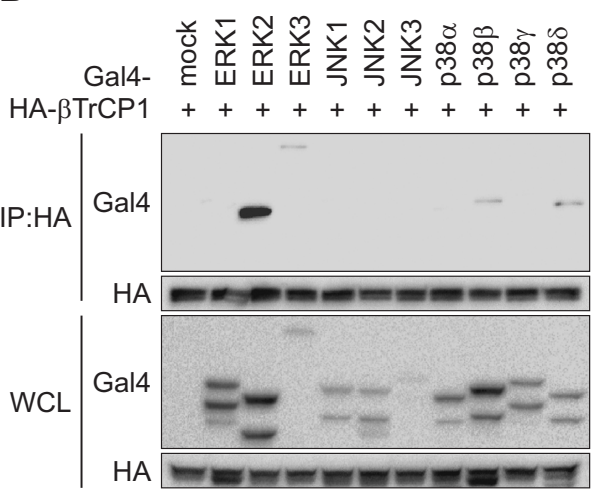

C

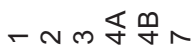

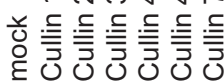

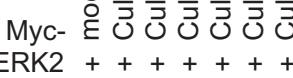

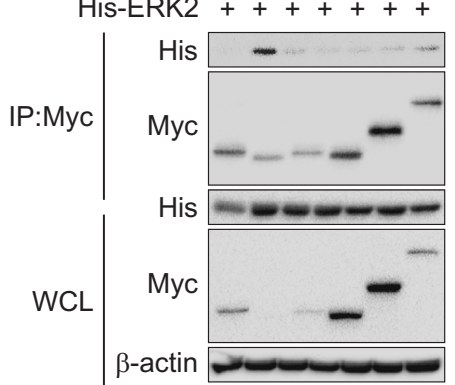

Figure 1. ERK2 and $\beta$ TrCP1 are components of the Cullin 1 containing SCF $^{\beta T r C P 1}$ complex. (A) Mammalian two-hybrid assay screening. Mammalian two-hybrid recombinant plasmids including pACT-VP16-b $\beta \operatorname{TrCP1}$, pG5-luciferase reporters, and each of pBIND-Gal4-kinases (as indicated) were transfected into HEK293T cells with a molar ratio 1:1:1. Relative luciferase activity was converted by comparison to luciferase activity obtained from pACT-VP16- $\beta$ TrCP1/pBIND-Gal4-mock/pG5-luc. Equal transfection was normalized by Renilla luciferase activity obtained from each of pBIND-Gal4-kinase expression vectors. (B) Confirmation of $\beta$ TrCP1 and ERK2 interaction obtained through IP. pBIND-Gla4-MAPKs (as indicated) and pcDNA3-HA- $\beta$ TrCP1 were co-transfected into HEK293T cells. The interaction of ERK2 and $\beta \operatorname{TrCP} 1$ was visualized by IP/Western blotting as indicated. (C) Confirmation of ERK2 and Cullin 1 interaction. pcDNA3-Myc-Cullins and pcDNA4-HisMAX-ERK2 were co-transfected into HEK293T cells. The interaction of ERK2 and each of the Cullins was visualized by IP/Western blotting as indicated. CDK, cyclin-dependent kinase; DAPK, death-associated protein kinase; GRK5, G protein-coupled receptor kinase 5; LCK, lymphocyte-specific protein-tyrosine kinase; NEK6, NIMA-related kinase 6; CSK, C-terminal SRC kinase; STK16, serine/threonine-protein kinase 16; TOPK, lymphokine-activated killer T-cell-originated protein kinase; PAK2, p21 protein-activated kinase 2; SGK, serum/glucocorticoid-regulated kinase; ERK, extracellular signal-activated kinase; JNK2, c-Jun N-terminal kinase 2; IP, immunoprecipitation; HA, hemagglutinin; WCL, whole cell lysates. 
were visualized by an enhanced chemiluminescence detection system (Amersham Biosciences, Piscataway, NJ, USA) using a Chemidoc $\mathrm{XRS}^{+}$imager (Bio-Rad Laboratories, Hercules, CA, USA).

\section{IP}

HEK293T cells $\left(2 \times 10^{6}\right)$ were seeded into $100-\mathrm{mm}$ dishes and incubated overnight. Individual expression vectors were transfected into HEK293T with jetPEI as indicated, and the cells were incubated for 24 hours. Protein samples from the cells were extracted by using a NP-40 cell lysis buffer. IP was conducted with the same amount of each sample and the Ab specific to the vector-transfected sample. The protein extracts were combined with protein $\mathrm{G}$ beads ( $50 \%$ slurry) (Cat. \#: 170618-02, Protein G Sepharose 4 Fast Flow, GE Healthcare, Little Chalfont, UK) by rocking at $4^{\circ} \mathrm{C}$ for at least 5 hours or overnight. The protein $\mathrm{G}$ beads were washed and mixed with $6 \times$ SDS sample buffer and boiled. The precipitated proteins were resolved by $10 \%$ to $15 \%$ SDS PAGE and detected by Western blotting using specific Abs as indicated.

\section{Statistical analysis and figure panels}

Mammalian two-hybrid screening data was obtained from a triplicated experiment. Data are expressed as mean \pm SEM values. Student's $t$-test using the Microsoft Excel program (Microsoft, Redmond, WA, USA) was used to compare values between two groups. $P$-values $<0.05$ (two-tailed) were considered significant. The Western blotting and IP experiments were conducted at least twice. The figure panels are representative photographs for Western blotting.

\section{RESULTS}

\section{ERK2 and $\beta$ TrCP1 are components of Cullin 1 SCF complex}

To identify the new binding partners to $\beta \operatorname{TrCP} 1$, we conducted a mammalian two-hybrid assay using kinases which were constructed in our laboratories. We found that mitogen-activated protein kinases (MAPK), including ERK2, ERK3, p38 $\alpha$, p38 , and JNK2, showed a relatively high interaction strength (about 3-5.4 folds) compared to other kinases, including CDK1 (cyclin-dependent kinase 1), CDK10, CHK1 (cell cycle

\begin{tabular}{|c|c|c|}
\hline Substrates & $\begin{array}{l}\text { MAPK docking } \\
\text { sequence }\end{array}$ & $\begin{array}{l}\text { docking } \\
\text { kinases }\end{array}$ \\
\hline MKP3 & IMLRRLQKGNLPV & ERK \\
\hline PTPN5 & LQERRGSNVSLTL & ERK \\
\hline DUSP8 & LVKRRLQQGKVTI & P38, JNK \\
\hline MEK1 & MPKKKP--TPIQL & ERK \\
\hline RSK1 & LAQRRVRK--LPS & ERK \\
\hline$\beta \operatorname{TrCP} 1$ & MLWKKLIERMVRT & N.D. \\
\hline$\beta \operatorname{TrCP} 1$ & LAERRGWGQYLFK & N.D. \\
\hline
\end{tabular}

MAPK docking sequence:

$(R / K)-(R / K)-X X X X X-(L / V / I)$
B

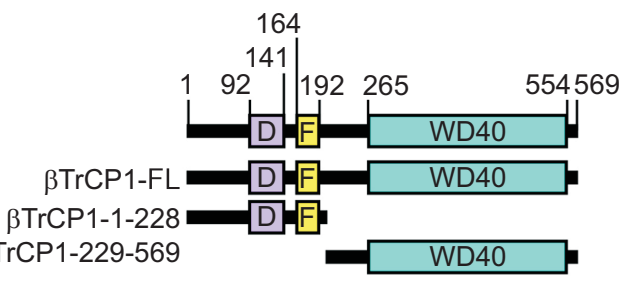

C

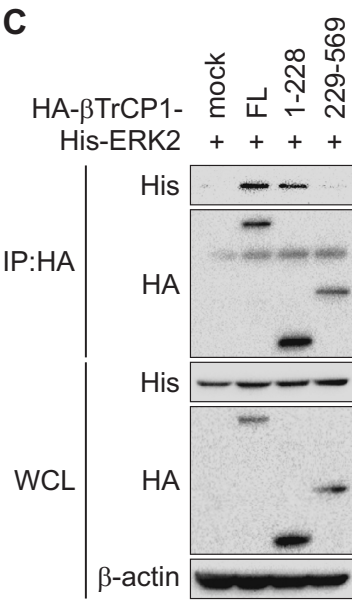

D

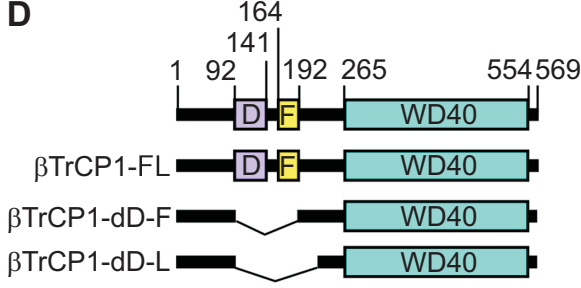

E

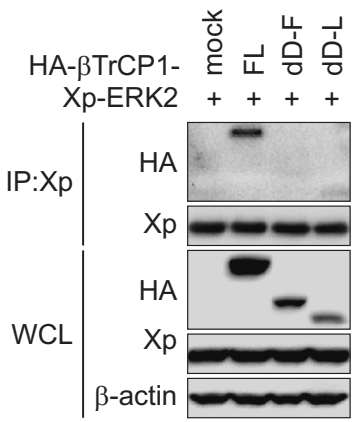

Figure 2. ERK2 binds to $F$ and linker domains of $\beta \operatorname{TrCP1}$. (A) Amino acid alignment of putative ERKs docking sites in $\beta \operatorname{TrCP1}$. MAPK docking sequence was noted in footnote. (B) Construction strategies for $\beta \operatorname{TrCP} 1$ truncated mutants. The mutants were recombined into pcDNA3-HA. (C) The constructs of $(B)$ were co-transfected as indicated. (D) Construction strategies for $\beta \operatorname{TrCP} 1$ intradomain deletion mutants. The mutants were recombined into pcDNA3-HA. (E) Interaction domain deciphering of $\beta \operatorname{TrCP} 1$ to ERK2. The constructs in (D) were co-transfected as indicated. (C, E) The interaction of ERK2 and each of $\beta \operatorname{TrCP} 1$ mutants were visualized by IP/Western blotting as indicated. MKP3, mitogen-activated protein kinase phosphatase 3; ERK, extracellular signal-regulated kinase; PTPN5, protein-tyrosine phosphatase nonreceptor type 5; DUSP8, dual-specificity phosphatase 8; MEK1, MAP kinase kinase 1; RSK1, ribosomal S6 kinase 1; N. D., not determined; MAPK, mitogen-activated protein kinases; D, D domain; F, F-box domian; FL, full length; IP, immunoprecipitation; HA, Hemagglutinin; WCL, whole cell lysates, Xp, Xpress. 
check kinase 1), DAPK2 (death-associated protein kinase 2), DAPK3, GRK5 (G protein-coupled receptor kinase 5), LCK (lymphocyte-specific protein-tyrosine kinase), NEK6 (NIMA-related kinase 6), CSK (C-terminal SRC kinase), STK16 (serine/threonine-protein kinase 16), TOPK (lymphokine-activated killer T-cell-originated protein kinase), PAK2 (p21 protein-activated kinase 2), SGK (serum/glucocorticoid-regulated kinase) (Fig. 1A). Since $\beta \operatorname{TrCP} 1$ showed a strong interaction with the MAPK members, IP was utilized to confirm binding to the following groups: ERK1, ERK2, ERK3, JNK1, JNK2, JNK3, p38 $\alpha$, p38 $\beta$, p38 $\gamma$, and p38 (Fig. 1B). The IP of $\beta T r C P 1$, using the HA tag $A b$, showed strong coprecipitation with ERK2 (Fig. 1B, upper panel of IP). ERK3, p38 $\beta$, and p38 $\delta$ bands coprecipitated with $\beta$ TrCP1 were weak compared to ERK2, which was attributable to the lower expression levels of these proteins in the whole cell lysates (WCL) (Fig. 1B, upper panel). Notably, since $\beta \operatorname{TrCP} 1$ has been classified as a member of the Cullin 1 containing SCF complex [13], ERK2 showed a band in IP with Cullin 1 when IP was conducted with Myc Ab (Fig. 1C, top panel). Additionally, the His-ERK2 protein levels in WCL were similar to other lanes in which were co-transfected with His-ERK2 and each of Myc-Cullin 2, 3, 4A, 4B, and 7 (Fig. 1C, $3^{\text {rd }}$ panel from top). These results indicate that ERK2 is a new interacting partner with $\beta \operatorname{TrCP} 1$.

\section{ERK2 binds to F-box and linker domains of $\beta \operatorname{TrCP} 1$}

After the discovery of the interaction between $\beta \operatorname{TrCP} 1$ and ERK2, the ERK2-binding domains of $\beta \operatorname{TrCP} 1$ were then determined. Since ERK2 is a member of MAPK family proteins, and ERKs docking consensus sequences are published as

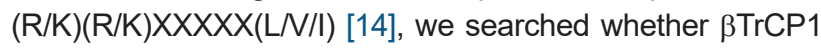
contained the amino acid sequences or not. Surprisingly, $\beta \operatorname{TrCP} 1$ harbored two consensus sequences in the F-box domain and the linker domain between the F-box and WD40 domains (Fig. 2A). The conserved KK-----V (referred to as PEDS1) and RR-----L (referred to as PEDS2) were located at amino acid 190 to 197 and 209 to 216 (Fig. 2A). To decipher more detail, we constructed $\beta \mathrm{TrCP} 1$ deletion mutant expression vectors using a pcDNA3-HA tag expression vector (Fig. 2B). The IP experiment using cell lysates expressing HisERK2 and each of HA-mock, $\beta$ TrCP1-FL, $\beta$ TrCP1-1-228, and $\beta$ TrCP1-229-569 showed that ERK2 was co-immunoprecipitated with $\beta \operatorname{TrCP} 1-\mathrm{FL}$ and $\beta \operatorname{TrCP} 1-1-228$ but not $\mathrm{HA}$ mock and $\beta$ TrCP1-229-569 (Fig. $2 \mathrm{C}$ ). To verify the interaction between aa 190-216 of $\beta$ TrCP1 and ERK2, we constructed $\beta \operatorname{TrCP} 1$ truncated mutant expression vectors: $\beta \operatorname{TrCP} 1-\mathrm{dD}-\mathrm{F}-$ box and $-d D-L$ (Fig. 2D). We confirmed that none of $\beta$ TrCP1 truncated proteins co-immunoprecipitated with ERK2, while HA- $\beta$ TrCP1-FL presented a strong co-immunoprecipitated band for ERK2 (Fig. 2E). Since the WD40 domain of $\beta$ TrCP1 is located in $\beta \operatorname{TrCP} 1-229-569$, these results suggested that ERK2 and $\beta$ TrCP1 binding may not trigger the ERK2 protein stability, but rather ERK2-mediated $\beta$ TrCP1 phosphorylation.
Determination of $\beta$ TrCP1 binding sites to ERK2 Our previous results indicated that PEDS1 and PEDS2 of $\beta$ TrCP1 located in the $F$ and linker domains played a key role in the interaction between $\beta \operatorname{TrCP} 1$ and ERK2. Since ERK 1 and 2 play a pivotal role in intreacellular signal transduction activated by diverse mitogenic stimuli that include growth factors [15,16], we hypothesized that PEDS1 and PEDS2 of $\beta$ TrCP1 might have been highly conserved in different species. Thus, we conducted comparative analysis of $\beta$ TrCP 1 in various species including mouse, rat, cow, monkey, snake, salmon, ant, and xenopus (Fig. 3A). Despite varying numbers of amino acids from the $\mathrm{N}$-terminus, it was found that amino acid sequences for the ERK docking sites were perfectly
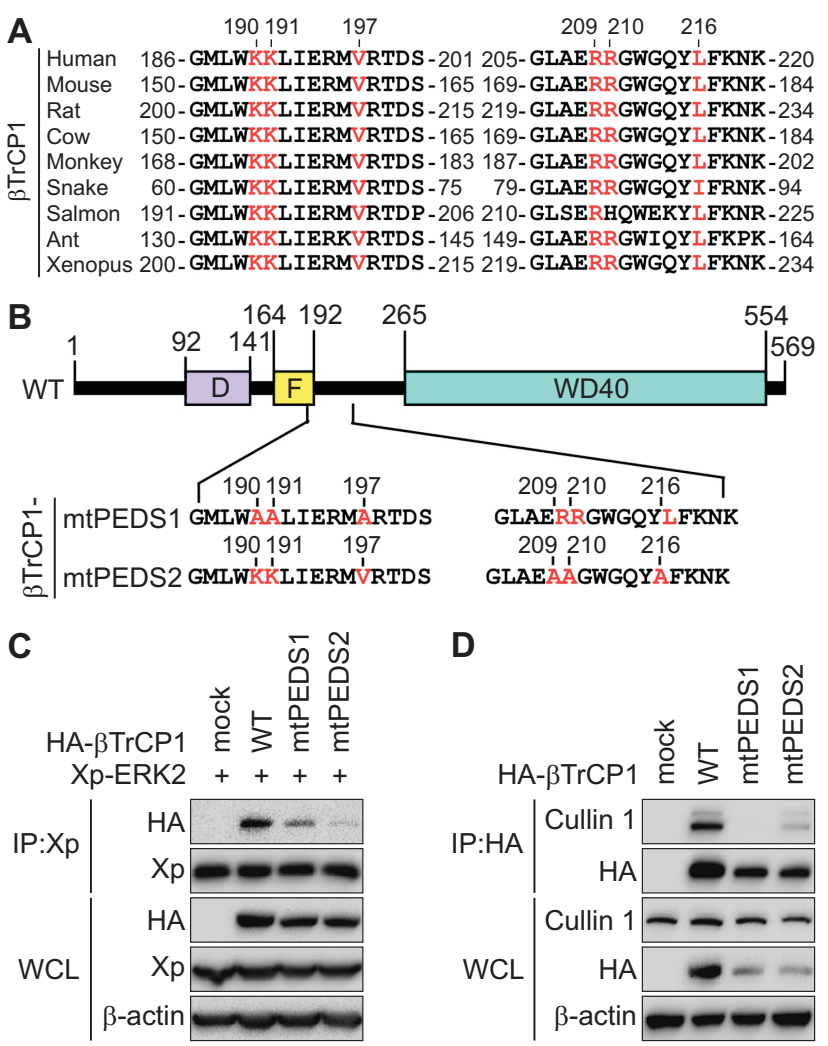

Figure 3. Determination of ERK2 binding sites of $\beta \operatorname{TrCP} 1$. (A) Amino acid homology of putative ERK2 docking sites (PEDS). Amino acids for two putative ERK2 docking sites were compared to different animal species as indicated. The numbers at the top indicate the position of amino acids. The red color indicates amino acids for putative ERK2 docking sites. (B) Mutation strategies to construct mutant PEDS 1 and 2. The mutants were recombined into pcDNA3-HA plasmids. (C) Confirmation of $\beta$ TrCP1 PEDS 1 and 2 for ERK2 binding. The pcDNA4HisMAX-ERK2 and each of the constructs in (B) were co-transfected as indicated. The interaction between ERK2 and each of $\beta$ TrCP1 mutants was visualized by IP/Western blotting as indicated. (D) The constructs in (B) were transfected into HEK293T cells. Endogenous Cullin 1 binding to $\beta \operatorname{TrCP} 1-\mathrm{mtPEDS} 1$ and -mtPEDS2 were visualized by IP/ Western blotting as indicated. WT, wildtype; D, D domain; F, F-box domain; mtPEDS, mutant putative ERK docking site; Xp, Xpress tag; ERK2, extracellular signal-regulated kinase 2; IP, immunoprecipitation; $\mathrm{HA}$, hemagglutinin; WCL, whole cell lysates. 
conserved in these animal species (Fig. 3A). To confirm whether the PEDS1 and PEDS2 played an essential role in the interaction with ERK2, we constructed two different mutants of $\beta \operatorname{TrCP} 1$ : $\beta \operatorname{TrCP} 1-m \mathrm{tPEDS} 1$ and $\beta \operatorname{TrCP} 1-m \mathrm{PPEDS} 2$. $\beta$ TrCP1-mtPEDS1 was constructed by replacing the lysines at aa 190 and 191 and valine at aa 197 to alanine and $\beta$ TrCP1-mtPEDS2 by replacing the arginines at aa 209 and 210 and leucine at aa 216 to alanine (Fig. 3B). The essential role of PEDS 1 and 2 of $\beta$ TrCP 1 in the interaction with ERK2 was proved by the IP using Xp-ERK2 and each of HA$\beta$ TrCP1-PEDS1 or -PEDS2. We found that ERK2 IP with Xp-tag Ab showed a dramatic decrease of both HA- $\beta$ TrCP1PEDS1 or -PEDS2 (Fig. 3C). Importantly, we further found that the HA- $\beta$ TrCP1-PEDS1 and -PEDS2 decreased the co-immunoprecipitated Cullin 1 (Fig. 3D). These results indicated that the amino acids, such as lysines at 190 and 191 , valine 197, arginines at 209 and 210 and leucine 216, play a role in not only ERK2 docking, but also Cullin 1-mediated SCF complex formation.

\section{ERK2-mediated $\beta$ TrCP1 phosphorylation affects $\beta$ TrCP1 stability}

Our previous results suggested that ERK2 and $\beta \operatorname{TrCP} 1$ interaction may involve ERK2-mediated $\beta$ TrCP1 phosphorylation. Since EGF-mediated signaling pathways induce ERK 1 and 2 phosphorylation and activation, we examined EGF-induced
A

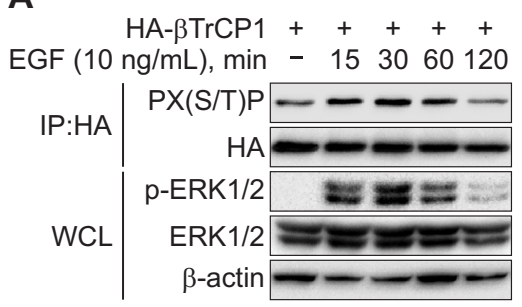

B

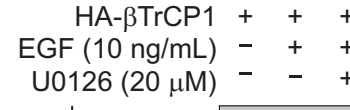
IP:HA $\begin{array}{r}\mathrm{PX}(\mathrm{S} / \mathrm{T}) \mathrm{P} \\ \mathrm{HA} \\ \mathrm{P}-\mathrm{ERK} 1 / 2= \\ \mathrm{ERK} 1 / 2= \\ \mathrm{HA} \\ \beta \text {-actin }\end{array}$
C<smiles>C=C</smiles>

D

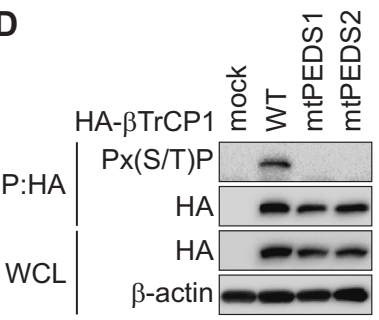

E

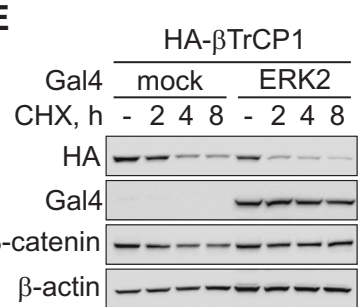

$\mathbf{F}$

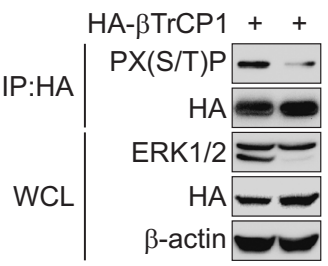

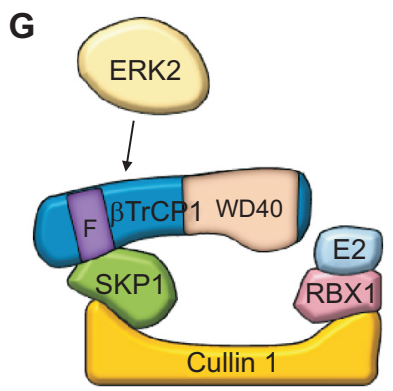

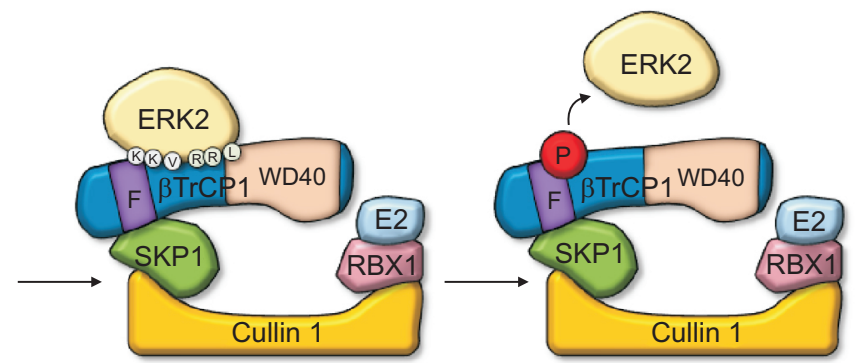

Figure 4. ERK2-mediated $\beta \operatorname{TrCP} 1$ phosphorylation affects $\beta \operatorname{TrCP} 1$ stability. (A) EGF stimulation induces $\beta \operatorname{TrCP} 1$ phosphorylation. The HEK293T cells transiently expressing HA- $\beta$ TrCP1 were starved, stimulated with EGF, and harvested as described in Materials and Methods. (B) ERKs Inhibition suppresses $\beta \operatorname{TrCP} 1$ phosphorylation. The HEK293T cells transiently expressing HA- $\beta$ TrCP1 were starved, stimulated with EGF or the EGF/MEK inhibitor U0126 as indicated, and harvested after 30 minutes. (C) ERK2 knockdown suppresses $\beta$ TrCP1 phosphorylation. HEK293T cells stably expressing sh-ERK2 cells were transfected with pcDNA3-HA- $\beta \operatorname{TrCP} 1$. (D) Disruption of $\beta \operatorname{TrCP} 1$ and ERK2 interaction prevents $\beta \operatorname{TrCP} 1$ phosphorylation. Cell lysates transiently expressing $\beta$ TrCP1 in HEK293T cells were utilized. (E) ERK2 reduces the half-life of $\beta$ TrCP1. The pcDNA3HA- $\beta \operatorname{TrCP} 1$ was co-transfected with pBIND-Gal4-mock or -ERK2 into HEK293T cells. The cells were treated with cycloheximide $(10 \mu \mathrm{g} / \mathrm{mL}) \mathrm{and}$ harvested at the indicated time points. The $\beta \operatorname{TrCP} 1$ protein levels were visualized by Western blotting as indicated. (F) ERK2 knockdown attenuates $\beta$ TrCP1 destabilization. HEK293T cells stably expressing sh-ERK2 cells were harvested at the indicated time point after $\mathrm{CHX}(10 \mu \mathrm{g} / \mathrm{mL})$ treatment. The proteins were visualized by Western blotting using specific antibodies as indicated. (G) ERK2 is a upstream kinase of $\beta \operatorname{TrCP} 1$, resulting in reduction of $\beta \operatorname{TrCP} 1$ half-life. (A-D) The phosphorylation of $\beta \operatorname{TrCP} 1$ was visualized by IP/Western blotting as indicated. $\beta$-actin was used for equal protein loading as an internal control. IP, immunoprecipitation; HA, hemagglutinin; ERK, extracellular signal-regulated kinase; WCL, whole cell lysates; CHX, cycloheximide; F, F-box domain. 
$\beta \operatorname{TrCP} 1$ phosphorylation. To verify $\beta \operatorname{TrCP} 1$ phosphorylation, we conducted IP using HA-tag Ab by combining the cell lysates transiently expressing $\mathrm{HA}-\beta \operatorname{TrCP} 1$ and stimulated with EGF. The phosphorylation levels of HA- $\beta$ TrCP1 were observed by Western blotting using phospho-serine/threonine $A b$. The results indicated that the HA- $\beta \operatorname{TrCP} 1$ phosphorylation level by EGF stimulation was increased at 15 minutes, sustained to 60 minutes, and decreased at 120 minutes (Fig. $4 \mathrm{~A})$. The induction pattern of HA- $\beta \operatorname{TrCP} 1$ phosphorylation was similar to ERK 1 and 2 phosphorylation by EGF stimulation (Fig. 4A). Interestingly, HA- $\beta$ TrCP1 total protein levels in whole cell lysates had an inverse correlation with ERK 1/2 phospho- and total-protein levels (Fig. 4A). Importantly, the MEKs inhibitor U0126 treatment completely blocked HA- $\beta$ TrCP1 phosphorylation induced by EGF stimulation (Fig. 4B). By Western blotting using the whole cell lysates, EGF-induced phosphorylation of ERK 1 and 2 disappeared with MEKs inhibitor U0126 treatment (Fig. 4B). Notably, genetic knockdown of ERK2 using pLenti-sh-ERK2 (Fig. 4C, $3^{\text {rd }}$ panel from top) suppressed phosphorylation of HA- $\beta$ TrCP1 (Fig. $4 \mathrm{C}$, top panel). The evidence indicating that ERK2 is an upstream kinase of $\beta \operatorname{TrCP} 1$ was provided by IP/Western blotting using cell lysates transiently expressing HA- $\beta$ TrCP1WT, HA- $\beta$ TrCP1-mtPEDS1 and HA- $\beta$ TrCP1-mtPEDS2 (Fig. $4 D)$. The results demonstrated that disruption of ERK2 and $\beta$ TrCP1 interaction blocked HA- $\beta$ TrCP1 phosphorylation (Fig. $4 D)$. Surprisingly, ectopic co-expression of ERK2 and $\beta$ TrCP1 reduced total protein levels and the half-life of $\mathrm{HA}-\beta \operatorname{TrCP} 1$ protein after cycloheximide treatment (Fig. 4E). In contrast, ERK2 knockdown using sh-ERK2 attenuated the $\beta \operatorname{TrCP} 1$ protein reduction, but not strong, by cycloheximide treatment (Fig. 4F). Taken together, these results indicate that ERK2 is an upstream kinase of $\beta \operatorname{TrCP} 1$ and growth factor-induced $\beta \operatorname{TrCP} 1$ phosphorylation by ERK2 reduces the half-life of $\beta \operatorname{TrCP} 1$ (Fig. 4G).

\section{DISCUSSION}

EGF-mediated signaling pathways are well-known oncogenic signaling pathways regulating cell proliferation, cell transformation, metastasis, and apoptosis [3,15]. Stimulation at the cytoplasmic membrane evokes activation signals and transduces the activation signal to the nucleus via a phosphate-delivery system. Since the members are stimulated by diverse mitogenic factors, including growth factors, serum, cytokines, hormones and environmental stresses, the members are referred to as MAPK, which consists of ERKs, p38 kinases, and JNK [16]. Although Ras proteins, upstream signaling molecules of ERKs, often show constitutively active mutations with high percentage in many human solid cancers [17], we were curious about why ERKs mutations have not been reported in human solid cancer. We hypothesized that 1) since ERKs play a pivotal role in cell survival, ERKs mutation may be lethal to the cells, resulting in spontaneous elimination 2) since ERKs' roles are backed up by other ERK isozymes, apparent phenotypes are not observable; and 3) since ERKs roles are trivial in biological processes, ERKs mutations may have not affected the manifestation of phenotypes. However, for the last several decades, research has emphasized the importance of ERK as a prime signaling molecule [16]. Recently, we found that ERK1 and 2 induce KIf4 phosphorylation, resulting in the formation of a $\mathrm{SCF}^{\beta T r C P}$ complex and Klf4 degradation [4]. Although there is no evidence that the SCF ${ }^{\beta T r C P}-\mathrm{KIf} 4$ complex contains ERK 1 or 2, it was found that ERK2 is a new binding partner of $\beta \operatorname{TrCP} 1$ (Fig. 1). Thus, ERK2 might affect the biological processes depending on not only the protein stability regulation, but also gene transcription.

The role of $\beta$ TrCP 1 and 2 are controversial. Since $\beta$ TrCPs mRNA and protein levels are increased in $56 \%$ of colorectal cancers and showed poor prognosis [10]. Moreover, hepatoblastoma [11] and some breast cancers [18] showed high expression of $\beta \operatorname{TrCP} 1$ and $\beta \operatorname{TrCP} 2$. NF- $\mathrm{kB}$ activation was observed in hepatocellular carcinoma [19]. Since the constitutively active $\mathrm{NF}-\kappa \mathrm{B}$ was associated with $\beta \mathrm{TrCP}$-mediated $\mathrm{I}_{\kappa} \mathrm{B}$ degradation [20], the signaling pathways produced by various cytokines, growth factors, and diverse stresses might be regulated by $\beta \operatorname{TrCP}$. In contrast, somatic mutations of $\beta \operatorname{TrCP} 1$ and $\beta$ TrCP2 that abolish E3 ligase activity were detected in human gastric cancer $[12,21]$. These mutations increase $\beta$-catenin stabilization in gastric cancer tissues [21]. Thus, the role of $\beta$ TrCPs is context-dependent as a tumor suppressor or oncogene. Since our research has demonstrated that RSK2, downstream of ERK 1 and 2, enhances NF-KB transactivation activity [22], ERK 1 and 2-mediated tumorigenesis might have a connection with $\beta$ TrCP-mediated protein stability regulation.

In this study, we found that $\beta$ TrCP1 and ERK2 interaction is mediated via $F$ and linker domains of $\beta \operatorname{TrcP} 1$, but not the WD40 domain (Fig. 2 and 3). Since substrates of F-box proteins generally interact with the WD40 domain, this point was crucial in hypothesizing that the interaction between $\beta$ TRCP1 and ERK2 was not aimed to degrade ERK2 proteins. However, since ERK2 phosphorylates $\beta$ TrCP1 (Fig. 4), the ERK2 and $\beta$ TrCP1 interaction might affect $\beta \operatorname{TrCP} 1$ enzymatic activity although the detailed mechanisms are unknown. We have considered a possible mechanism. Phosphorylation plays a key role in protein-protein interaction as well as protein degradation, especially in ubiquitin-proteasome systems [23]. In fact, many proteins such as c-Myc, cyclin E, c-Jun, Notch1 and androgen receptor are degraded by E3 ubiquitin ligase after phosphorylation by specific kinases, respectively [24-27]. Although we did not identify specific E3 ligase that regulates the protein stability of $\beta \operatorname{TrCP} 1$, we found that ERK2 could phosphorylate $\beta \operatorname{TrCP} 1$ and that the mutants of $\beta \operatorname{TrCP} 1$ (mtPEDS1 and mtPEDS2) decreased the interaction with ERK2 (Fig. 3C). Furthermore, overexpression of ERK2 reduced $\beta$ TrCP1 protein half-life under cycloheximide treatment 
(Fig. 4E). Therefore, we suggest that protein stability of the mutants of $\beta$ TrCP1 (mtPEDS1 and mtPEDS2) would be increased compared to the $\beta \operatorname{TrCP} 1$ wild type.

The $p 90^{\mathrm{RSK}}$ family members RSK1-4 and MSK1-2, regulatory kinases located between ERKs and many transcription factors in the signaling pathway, are well-known substrates of ERK1 and ERK2 which play essential roles in diverse cellular processes [28]. Since at least 200 ERK substrates have been discovered, ERK1- and ERK2-mediated cellular signaling might be dependent on the subcellular distribution of ERKs and their substrates and stimulus-dependent signaling axis activation by protein-protein interaction [16,28]. As ERK2 being a proline-directed kinase, the phosphorylation of ERKs substrates occurs at Pro-X-Ser/Thr-Pro consensus sequences [29]. In this study, we found that $\beta \operatorname{TrCP} 1$ contained two independent PEDSs; however, the phosphorylation site(s) by ERK2 is still unknown.

\section{ACKNOWLEDGMENTS}

This research was funded by the Ministry of Science, ICT and Future Planning (NRF-2020R1A2B5B02001804 and NRF2020R1A4A2002894), and the Ministry of Education (BK214th-sponsored Advanced Program for SmartPharma Leaders 4299990814607).

\section{CONFLICTS OF INTEREST}

No potential conflicts of interest were disclosed.

\section{ORCID}

Cheol-Jung Lee, https://orcid.org/0000-0002-0322-2018

Ga-Eun Lee, https://orcid.org/0000-0001-8700-209X

Hyun-Jung An, https://orcid.org/0000-0002-3189-7944

Eun Suh Cho, https://orcid.org/0000-0001-5373-2916

Weidong Chen, https://orcid.org/0000-0002-4015-6005

Joo Young Lee, https://orcid.org/0000-0002-6020-3040

Han Chang Kang, https://orcid.org/0000-0003-0696-1155

Hye Suk Lee, https://orcid.org/0000-0003-1055-9628

Yong-Yeon Cho, https://orcid.org/0000-0003-1107-2651

\section{REFERENCES}

1. Hubbard SR, Miller WT. Receptor tyrosine kinases: mechanisms of activation and signaling. Curr Opin Cell Biol 2007;19:117-23.

2. Cullen PJ, Lockyer PJ. Integration of calcium and Ras signalling. Nat Rev Mol Cell Biol 2002;3:339-48.

3. Arul N, Cho YY. A rising cancer prevention target of RSK2 in human skin cancer. Front Oncol 2013;3:201.

4. Kim MO, Kim SH, Cho YY, Nadas J, Jeong CH, Yao K, et al. ERK1 and ERK2 regulate embryonic stem cell self-renewal through phosphorylation of Klf4. Nat Struct Mol Biol 2012;19:28390.
5. Yoon S, Seger R. The extracellular signal-regulated kinase: multiple substrates regulate diverse cellular functions. Growth Factors 2006;24:21-44.

6. Maik-Rachline G, Seger R. The ERK cascade inhibitors: towards overcoming resistance. Drug Resist Updat 2016;25:1-12.

7. Wang Z, Liu P, Inuzuka H, Wei W. Roles of F-box proteins in cancer. Nat Rev Cancer 2014;14:233-47.

8. Lee CJ, An HJ, Kim SM, Yoo SM, Park J, Lee GE, et al. FBXW7mediated stability regulation of signal transducer and activator of transcription 2 in melanoma formation. Proc Natl Acad Sci USA 2020;117:584-94.

9. Kudo Y, Guardavaccaro D, Santamaria PG, Koyama-Nasu $\mathrm{R}$, Latres $\mathrm{E}$, Bronson $\mathrm{R}$, et al. Role of $\mathrm{F}$-box protein $\beta \operatorname{Trcp} 1$ in mammary gland development and tumorigenesis. Mol Cell Biol 2004;24:8184-94.

10. Ougolkov A, Zhang B, Yamashita K, Bilim V, Mai M, Fuchs SY, et al. Associations among $\beta$-TrCP, an E3 ubiquitin ligase receptor, $\beta$-catenin, and NF-kB in colorectal cancer. J Natl Cancer Inst 2004;96:1161-70.

11. Koch A, Waha A, Hartmann W, Hrychyk A, Schüller U, Waha A, et al. Elevated expression of Wnt antagonists is a common event in hepatoblastomas. Clin Cancer Res 2005;11:4295-304.

12. Kim CJ, Song JH, Cho YG, Kim YS, Kim SY, Nam SW, et al. Somatic mutations of the $\beta$-TrCP gene in gastric cancer. APMIS 2007;115:127-33.

13. Jin J, Cardozo T, Lovering RC, Elledge SJ, Pagano M, Harper JW. Systematic analysis and nomenclature of mammalian F-box proteins. Genes Dev 2004;18:2573-80.

14. Smith JA, Poteet-Smith CE, Malarkey K, Sturgill TW. Identification of an extracellular signal-regulated kinase (ERK) docking site in ribosomal S6 kinase, a sequence critical for activation by ERK in vivo. J Biol Chem 1999;274:2893-8.

15. Cho YY, Yao K, Kim HG, Kang BS, Zheng D, Bode AM, et al. Ribosomal S6 kinase 2 is a key regulator in tumor promoter induced cell transformation. Cancer Res 2007;67:8104-12.

16. Cho YY. Molecular targeting of ERKs/RSK2 signaling in cancers. Curr Pharm Des 2017;23:4247-58.

17. Prior IA, Hood FE, Hartley JL. The frequency of Ras mutations in cancer. Cancer Res 2020;80:2969-74.

18. Spiegelman VS, Tang W, Chan AM, Igarashi M, Aaronson SA, Sassoon DA, et al. Induction of homologue of Slimb ubiquitin ligase receptor by mitogen signaling. J Biol Chem 2002;277:36624-30.

19. Arsura M, Cavin LG. Nuclear factor- $\kappa B$ and liver carcinogenesis. Cancer Lett 2005;229:157-69.

20. Shirane M, Hatakeyama S, Hattori K, Nakayama K, Nakayama K. Common pathway for the ubiquitination of $I_{\kappa} B \alpha, I_{\kappa} B \beta$, and $I_{\kappa} B \varepsilon$ mediated by the F-box protein FWD1. J Biol Chem 1999;274:28169-74.

21. Saitoh $T$, Katoh M. Expression profiles of $\beta \operatorname{Trcp} 1$ and $\beta T R C P 2$, and mutation analysis of $\beta$ TRCP2 in gastric cancer. Int $\mathrm{J}$ Oncol 2001;18:959-64.

22. Lee CJ, Lee MH, Yoo SM, Choi KI, Song JH, Jang JH, et al. Magnolin inhibits cell migration and invasion by targeting the 
ERKs/RSK2 signaling pathway. BMC Cancer 2015;15:576.

23. Swaney DL, Beltrao P, Starita L, Guo A, Rush J, Fields S, et al. Global analysis of phosphorylation and ubiquitylation cross-talk in protein degradation. Nat Methods 2013;10:676-82.

24. Koepp DM, Schaefer LK, Ye X, Keyomarsi K, Chu C, Harper JW, et al. Phosphorylation-dependent ubiquitination of cyclin $\mathrm{E}$ by the SCFFbw7 ubiquitin ligase. Science 2001;294:173-7.

25. Welcker M, Orian A, Grim JE, Eisenman RN, Clurman BE. A nucleolar isoform of the Fbw7 ubiquitin ligase regulates C-Myc and cell size. Curr Biol 2004;14:1852-7.

26. Wei W, Jin J, Schlisio S, Harper JW, Kaelin WG Jr. The v-Jun point mutation allows c-Jun to escape GSK3-dependent recognition and destruction by the Fbw7 ubiquitin ligase. Cancer Cell 2005;8:25-33.

27. Ntziachristos P, Lim JS, Sage J, Aifantis I. From fly wings to targeted cancer therapies: a centennial for notch signaling. Cancer Cell 2014;25:318-34.

28. Cho YY. RSK2 and its binding partners in cell proliferation, transformation and cancer development. Arch Pharm Res 2017;40:291-303.

29. Sharrocks AD, Yang SH, Galanis A. Docking domains and substrate-specificity determination for MAP kinases. Trends Biochem Sci 2000;25:448-53. 